\title{
ASYMPTOTICS FOR THE EIGENVALUES OF A FOURTH ORDER DIFFERENTIAL OPERATOR IN A "DEGENERATE" CASE
}

\author{
KH.K. ISHKIN, KH. KH. MURTAZIN
}

\begin{abstract}
In the paper we consider the operator $L$ in $L^{2}[0,+\infty)$ generated by the differential expression $\mathcal{L}(y)=y^{(4)}-2\left(p(x) y^{\prime}\right)^{\prime}+q(x) y$ and boundary conditions $y(0)=$ $y^{\prime \prime}(0)=0$ in the "degenerate" case, when the roots of associated characteristic equation has different growth rate at the infinity. Assuming a power growth for functions $p$ and $q$, under some additional conditions of smoothness and regularity kind, we obtain an asymptotic equation for the spectrum allowing us to write out several first terms in the asymptotic expansion for the eigenvalues of the operator $L$.
\end{abstract}

Keywords: differential operators, asymptotics of spectrum, turning point.

Mathematics Subject Classification: 47E05, 34L16, 34L20, 34L40, 34B40

\section{INTRODUCTION}

The features of the spectral problems for ordinary differential operators allows one to employ one of the most effective methods based on asymptotic estimates for a fundamental system of solutions (FSS) of the equation

$$
\mathcal{L} y=\lambda y,
$$

(see, for instance, [1, 2]). For example, if $L$ is some self-adjoint extension of the minimal operator generated by the differential expression $\mathcal{L} y$ in $L^{2}(a, b)$ [1] and it has a discrete spectrum with the counting function $N(r)$, in order to study the asymptotics of $N(r)$ (as $r \rightarrow+\infty$ ), one can employ the Tauberian technique, but first WKB-estimates [3, Ch. III, Sect. 2] for kernel of the resolvent $(L-\lambda)^{-1}$ should be obtained for large $\lambda$ far from the spectrum of $L$. This kernel is expressed in terms of FSS for equation (1). But if we need to find several first terms in the asymptotics for the eigenvalues $\lambda_{n}$ taken in the ascending order counting multiplicities as $n \rightarrow+\infty$, then the Tauberian technique can not be applied and one has to "descend" to the spectrum and to study the asymptotics of the solutions to equation (1) as $\lambda$ goes to infinity along a set containing the spectrum of $L$ or a part of it. In the case, when the operator $L$ is singular [1], this circumstance, as a rule, gives rise to the turning points [3, Ch. III, Sect. 1]; in their vicinities the WKB-estimates do not work anymore. The method of pattern equations (Langer method) [4] allows one to obtain an approximate solution to equation (1) suitable both in the vicinity of the turning point and far from this point becoming the WKB-solution. This method is both effective for self-adjoint and non-self-adjoint spectral problems [5]. At present, the spectral problems with a turning point are quite well studied for two-terms operators [6][11].

Kh.K. Ishkin, Kh. Kh. Murtazin, Asymptotics for the eigenvalues of a fourth order DifferENTIAL OPERATOR IN A "DEGENERATE" CASE.

(c) Ishkin Kh.K., Murtazin Kh. Kh. 2016.

The work is supported by the Ministery of Education and Science of Russian Federation (grant no. 01201456408) and by RFBR (grant no. 15-01-01095).

Submitted June 15, 2016. 
In the paper we consider the operator $L$ in $L^{2}[0,+\infty)$ generated by the differential expression

$$
\mathcal{L}(y)=y^{(4)}-2\left(p(x) y^{\prime}\right)^{\prime}+q(x) y
$$

and the boundary conditions

$$
y(0)=y^{\prime \prime}(0)=0
$$

in the "degenerate" case, when the roots of the associated characteristic equation have different growth rate at infinity [2, Ch. IX, Sect. 4]. Assuming a power growth for the functions $p$ and $q$, under some additional smoothness and regularity assumptions we obtain the asymptotic equation for the spectrum, which allows us to write several terms in the asymptotic series for the eigenvalues $L$.

\section{PRELIMINARY REMARKS}

2.1. Main condition for the coefficients. We impose the following conditions for the real-valued functions $p$ and $q$ :

1. As $x \geqslant x_{0}\left(x_{0} \geqslant 0\right.$ is a constant), the functions $p$ and $q$ have absolutely continuous derivatives satisfying the inequalities

$$
a_{1} x^{\alpha-1} \leqslant q^{\prime}(x) \leqslant A_{1} x^{\alpha-1}, \quad b_{1} x^{\beta-1} \leqslant p^{\prime}(x) \leqslant B_{1} x^{\beta-1},
$$

where $a_{1}, A_{1}, b_{1}, B_{1}, \alpha, \beta$ are positive constants and

$$
\alpha<2 \beta \text {; }
$$

the second derivatives of the functions $p$ and $q$ are sign-definite almost everywhere.

2. The functions $p$ and $q$ are summable on $\left[0, x_{0}\right]$.

Remark 1. It follows from equation (4) that as $x \geqslant x_{1}\left(x_{1} \geqslant x_{0}\right)$,

$$
a x^{\alpha} \leqslant q(x) \leqslant A x^{\alpha}, \quad b x^{\beta} \leqslant p(x) \leqslant B x^{\beta},
$$

where $a, A, b, B$ are positive constants. Therefore [1, Sect. 24, Thm. 2], the spectrum of each self-adjoint extension of the minimal operator generated by expression (2) is discrete.

In what follows, under additional restrictions for the functions $p$ and $q$, we obtain the double asymptotics [3, Ch. 2, Sect. 7] of solutions to the equation $l(y)=\lambda y$, which, in particular, implies that the deficiency indices of the operator $L_{0}$ are equal to $(2,2)$. The latter fact yields the self-adjointness of the operator $L$.

Remark 2. The conditions under which the deficiency indices of the minimal operator generated by expression (2) are equal to (2, 2) were studied by many authors [12] - 18].

2.2. Reduction of the main equation to the canonical form. We introduce the notations. Let $\chi(x)$ be an infinitely differentiable function equalling to one on $\left[0, x_{0}\right]$ and vanishing on $\left[x_{0}+1, \infty\right)$. We let

$$
\begin{aligned}
& p_{1}(x)=p(x)(1-\chi(x)), \quad q_{1}(x)=q(x)(1-\chi(x)), \quad f(x, \lambda, \mu)=\mu^{4}-2 p_{1} \mu^{2}+q_{1}-\lambda, \\
& A_{1}=\left(\begin{array}{cccc}
0 & 1 & 0 & 0 \\
0 & 0 & 1 & 0 \\
0 & 2 p_{1} & 0 & -1 \\
q_{1}-\lambda & 0 & 0 & 0
\end{array}\right), \quad A_{2}=\chi\left(\begin{array}{cccc}
0 & 0 & 0 & 0 \\
0 & 0 & 0 & 0 \\
0 & 2 p & 0 & 0 \\
q & 0 & 0 & 0
\end{array}\right) .
\end{aligned}
$$

Let $Y=\left(y, y^{[1]}, y^{[2]}, y^{[3]}\right)^{T}$, where $y^{[k]}$ stands for the $k$ th quasi-derivative [1, Ch. V, Sect. 15]. Then the equation $\mathcal{L} y=\lambda y$ is equivalent to the system of the equations

$$
Y^{\prime}=\left(A_{1}+A_{2}\right) Y \text {. }
$$

The characteristic polynomial of the matrix $A_{1}$ coincides with the function $f(x, \lambda, \mu)$. The roots of the equation $f(x, \lambda, \mu)=0$ form two pairs

$$
\mu_{1,2}= \pm \sqrt{\nu_{1}}, \quad \mu_{3,4}= \pm \sqrt{\nu_{2}}
$$


where $\nu_{1,2}=p_{1} \pm \sqrt{D}, D=p_{1}^{2}+\lambda-q_{1}$, the branch of the root $\sqrt{z}$ is chosen so that $\sqrt{z}>0$ as $z>0$. Since $\nu_{2}=\left(q_{1}-\lambda\right) / \nu_{1}$, it follows from inequalities (4) and (5) that for each fixed $\lambda>0$ and for each $1 \leqslant i, j \leqslant 2$

$$
\mu_{j+2}=o\left(\mu_{i}\right), \quad x \rightarrow \infty
$$

that is, the "degenerate" case holds true.

Hereafter we assume that $\beta<\alpha+2$.

We introduce the matrices

$$
\begin{aligned}
& A_{0}=\operatorname{diag}\left(A_{01}, A_{02}\right), \\
& A_{01}=\sqrt{\nu_{1}} \operatorname{diag}(1,-1), \quad A_{02}=\left(\begin{array}{cc}
0 & 1 \\
\nu_{2} & 0
\end{array}\right), \\
& T=D^{-1 / 4}\left(\begin{array}{cc}
I_{2} & I_{2} \\
\Lambda_{1} & \Lambda_{2}
\end{array}\right) \operatorname{diag}\left(M W, I_{2}\right),
\end{aligned}
$$

$I_{2}$ is the unit matrix of second order,

$$
\begin{array}{ll}
\Lambda_{1}=\operatorname{diag}\left(\nu_{1},-\nu_{2}\right), & \Lambda_{2}=\operatorname{diag}\left(\nu_{2},-\nu_{1}\right), \\
W=\left(\begin{array}{cc}
1 & 1 \\
1 & -1
\end{array}\right), & M_{1}=\operatorname{diag}\left(\nu_{1}^{-1 / 4}, \nu_{1}^{1 / 4}\right), \\
B_{1}=-T^{-1} T^{\prime}, & B_{2}=T^{-1} A_{2} T .
\end{array}
$$

The entries of the matrix $B_{1}$ can be easily written:

$$
\begin{array}{ll}
B_{1}=\left(\begin{array}{cc}
B_{11} & B_{12} \\
B_{21} & B_{22}
\end{array}\right), & \\
B_{11}=\left(\begin{array}{cc}
0 & b_{1} \\
b_{1} & 0
\end{array}\right), & B_{12}=W^{-1} \operatorname{diag}\left(b_{2}, b_{3}\right), \\
B_{21}=\operatorname{diag}\left(b_{3}, b_{2}\right) W, & B_{22}=\operatorname{diag}\left(b_{4},-b_{4}\right), \\
b_{1}=-\frac{\nu_{1}^{\prime}}{4 \nu_{1}}-\frac{p^{\prime}}{2 \sqrt{D}}, & b_{2}=-\frac{\nu_{1}^{1 / 4} \nu_{2}^{\prime}}{2 \sqrt{D}}, \\
b_{3}=\frac{\nu_{1}^{\prime}}{2 \sqrt{D} \nu_{1}^{1 / 4}}, & b_{4}=\frac{p^{\prime}}{2 \sqrt{D}} .
\end{array}
$$

Let

$$
\begin{aligned}
& X=\left(\begin{array}{ll}
X_{11} & X_{12} \\
X_{21} & X_{22}
\end{array}\right), \\
& X_{11}=-\frac{1}{2} A_{01}^{-1} B_{11}, \\
& X_{12}=-\frac{1}{2 \sqrt{D}}\left(A_{01}\left(B_{12}+B_{12} A_{02}\right),\right. \\
& X_{21}=-\frac{1}{2 \sqrt{D}}\left(B_{21} A_{01}+A_{02} B_{21}\right), \\
& X_{22}=\left(\begin{array}{cc}
0 & 0 \\
-b_{4} & 0
\end{array}\right) .
\end{aligned}
$$

One can check easily the relations

$$
T^{-1} A T=A_{0}, \quad X A_{0}-A_{0} X=B_{1} .
$$

Then the substitution

$$
Y=T\left(I_{4}+X\right) V
$$


transforms equation (7) to the form

$$
V^{\prime}=\left(A_{0}+Z_{1}\right) V
$$

where

$$
Z_{1}=\left(I_{4}+X\right)^{-1}\left(B_{1} X-X^{\prime}+B_{2}\left(I_{4}+X\right)\right)
$$

\section{EqUATION FOR THE SPECTRUM}

3.1. Formulation of the main result. We introduce the notations:

$$
\begin{aligned}
& \xi(x, \lambda)=\left.\left.\left|\frac{3}{2} \int_{a_{\lambda}}^{x}\right| \nu_{2}\right|^{1 / 2} d t\right|^{2 / 3} \operatorname{sgn}\left(x-a_{\lambda}\right), \\
& S=\left(\xi^{\prime}(x, \lambda)\right)^{-1 / 2}, \quad K(x, \lambda)=\frac{S^{\prime \prime}}{S}, \\
& \widetilde{K}(t, \lambda)=a_{\lambda}^{2}\left[\left|K\left(a_{\lambda} t, \lambda\right)\right|+\left(\frac{\left|p_{1}^{\prime \prime}\right|+\left|q_{1}^{\prime \prime}\right|}{\sqrt{D}}\right)\left(a_{\lambda} t, \lambda\right)\right] ;
\end{aligned}
$$

$a_{\lambda}$ is the root of the equation $q\left(a_{\lambda}\right)=\lambda$.

The main result of this section is the following theorem.

Theorem 1. Assume that for $\beta<\alpha+2$, Conditions 1), 2) hold and moreover,

3. The function $\widetilde{K}(t, \lambda)$ is bounded in some vicinity of the point $t=1$ of the form $(1-\delta, 1+\delta)$ $(\delta>0$ is independent of $\lambda)$ uniformly in $\lambda \geqslant \Lambda_{0}, \Lambda_{0}>0$ is a constant.

Then the eigenvalues of the operator $L$ are determined by the equation

$$
\sin \Phi(\lambda)+K(\lambda) \cos \Phi(\lambda)+O\left(b(\lambda)+\lambda^{-\delta}\right)=0,
$$

where

$$
\begin{aligned}
& \Phi(\lambda)=\int_{0}^{a_{\lambda}}\left|\nu_{2}(t, \lambda)\right|^{1 / 2} d t+\frac{\pi}{4}, \\
& K(\lambda)=-\frac{5}{72}\left(\Phi(\lambda)-\frac{\pi}{4}\right)^{-1}+\frac{1}{2} \int_{0}^{a_{\lambda}}\left|\nu_{2}\right|^{-1 / 2}\left(b_{4}^{2}+b_{4}^{\prime}-K(t, \lambda)+\frac{\nu_{2}^{\prime}\left(\nu_{2} \nu_{1}^{\prime}-\nu_{1} \nu_{2}^{\prime}\right)}{8 D^{3 / 2}}\right) d t \\
& b(\lambda)=\int_{0}^{a_{\lambda}}\left|\nu_{2}\right|^{-1 / 2} \exp \left(i \int_{0}^{t}\left|\nu_{2}\right|^{1 / 2} d t\right)\left[\frac{\left(p_{1}^{\prime}\right)^{2}+\left|q_{1}^{\prime \prime}\right|}{D}+\frac{\left|p_{1}^{\prime \prime}\right|}{\sqrt{D}}+|\chi||p|\right] d t, \\
& \delta=\min \left\{\frac{1}{2}, \frac{\alpha+2-\beta}{\alpha}, \frac{\alpha+2-\beta}{3 \alpha}+\frac{1}{\alpha}\right\} .
\end{aligned}
$$

If the function $p$ has an absolutely continuous derivative on the entire half-line $[0, \infty)$, then the number $1 / 2$ in the definition of $\delta$ can be replaced by $3 / 2$, while in the integral $b(\lambda)$ the term $|\chi p|$ can be replaced by 0 .

\subsection{Pattern equation. Let}

$$
Q_{1}(x, \lambda)=\int_{0}^{x} \nu_{1}^{1 / 2} d t, \quad Q_{2}(x, \lambda)=\int_{a_{\lambda}}^{x}\left|\nu_{2}\right|^{1 / 2} d t .
$$

We choose pattern equations as

$$
\begin{aligned}
& V_{0}=\operatorname{diag}\left(V_{01}, V_{02}\right), \\
& V_{01}=\exp \left(\operatorname{diag}\left(Q_{1},-Q_{1}\right)\right), \\
& V_{02}=\left(\begin{array}{ll}
v_{1} & v_{2} \\
v_{1}^{\prime} & v_{2}^{\prime}
\end{array}\right), \\
& v_{1}=B v(\xi(x, \lambda)), \quad v_{2}=B u(\xi(x, \lambda)),
\end{aligned}
$$

where $v(\xi), u(\xi)$ are real Airy functions [19, Sect. 7.4.3]. 
It is easy to check that $V_{0}$ satisfies the equation

$$
\begin{aligned}
& V_{0}^{\prime}=A_{0} V_{0}+Z_{2} V_{0}, \\
& Z_{2}=\operatorname{diag}\left(0, \frac{S^{\prime \prime}}{S} J_{0}\right),
\end{aligned}
$$

where

$$
J_{0}=\left(\begin{array}{cc}
0 & 0 \\
1 & 0
\end{array}\right)
$$

0 is the zero matrix of second order.

We introduce the notations

$$
\begin{aligned}
& J=\operatorname{diag}(1,-1), \\
& d(x, \lambda)= \begin{cases}1, & x \geqslant a_{\lambda}, \\
0, & x<a_{\lambda},\end{cases} \\
& D(x, \lambda)=\exp \left[\operatorname{diag}\left(Q_{1} J,-d Q_{2} J\right)\right], \\
& T_{0}(x, \lambda)=\operatorname{diag}\left(1,1,\left|\nu_{2}\right|^{-\sigma(x, \lambda) / 4},\left|\nu_{2}\right|^{\sigma(x, \lambda) / 4}\right),
\end{aligned}
$$

where $\sigma(x, \lambda)$ is the characteristic function of a set, $[0, \infty),\left(a_{\lambda}\left(1-\delta_{1}\right), a_{\lambda}\left(1+\delta_{2}\right)\right), \delta_{1}, \delta_{2}$ are determined by the relations

$$
-Q_{2}\left(a_{\lambda}\left(1-\delta_{1}\right), \lambda\right)=Q_{2}\left(a_{\lambda}\left(1+\delta_{2}\right), \lambda\right)=1 .
$$

We let

$$
\widetilde{V}_{0}(x, \lambda)=T_{0}^{-1} V_{0} D^{-1} .
$$

It follows from the asymptotic formulae for the Airy functions [3, Ch. 4, Sect. 1] that

$$
\begin{aligned}
& \widetilde{V_{0}}=\operatorname{diag}\left(I_{2}, \widetilde{V_{02}}\right), \\
& \widetilde{V_{02}}=\left(\begin{array}{rr}
1 / 2 & 1 \\
-1 / 2 & 1
\end{array}\right)\left[I_{2}+O\left(Q_{2}^{-1}\right)+O\left(\nu_{2}^{\prime} \nu_{2}^{-3 / 2}\right)\right], \quad Q_{2} \rightarrow+\infty, \\
&\left.\widetilde{V_{02}=} \begin{array}{cc}
\sin \Phi & \cos \Phi \\
-\cos \Phi & \sin \Phi
\end{array}\right)\left[I_{2}-\frac{5}{72} Q_{2}^{-1}\left(\begin{array}{cc}
0 & 1 \\
-1 & 0
\end{array}\right)\right. \\
&\left.+O\left(Q_{2}^{-2}\right)+O\left(\nu_{2}^{\prime}\left|\nu_{2}\right|^{-3 / 2}\right)\right], \quad Q_{2} \rightarrow-\infty, \\
& \Phi=-Q_{2}(x, \lambda)+\frac{\pi}{4} .
\end{aligned}
$$

3.3. Integral equation. Applying the method of variation of constants, for FSS of system (15) we obtain the equation

$$
V=V_{0}(x, \lambda)+\int_{\Gamma(x)} V_{0}(x, \lambda) V_{0}^{-1}(t, \lambda) Z(t, \lambda) V(t, \lambda) d t
$$

where

$$
Z=Z_{1}-Z_{2}
$$

$\Gamma(x)$ is the matrix of the integration intervals $\gamma_{i j}(x)=\left(\gamma_{i j}, x\right), 0 \leqslant \gamma_{i j} \leqslant \infty$; the meaning is that each entry $u_{i j}$ of matrix $U:=V_{0}^{-1} Z V$ is integrated over its own interval $\gamma_{i j}(x)$ in the direction from $\gamma_{i j}$ to $x$. Let us show that under an appropriate choice of constants $\gamma_{i j}$, we can apply the successive approximations method to equation (28) that will allows us to construct the FSS of equation (15) with a known asymptotics for small $\lambda>0$ uniformly in $x \geqslant 0$.

We let

$$
\widetilde{V}=T_{0}^{-1} V D \text {. }
$$


Then $\widetilde{V}$ satisfies the equation

$$
\widetilde{V}=\widetilde{V}_{0}+A(\lambda) \widetilde{V}
$$

where

$$
\begin{aligned}
& (A(\lambda) \widetilde{V})(x, \lambda)=\widetilde{V}_{0}(x, \lambda) \int_{\Gamma(x)} A(x, t, \lambda)(\widetilde{V} D)(t, \lambda) D^{-1}(x, \lambda) d t \equiv \widetilde{V}_{0}(x, \lambda) A_{1}(\lambda) \widetilde{V}, \\
& A(x, t, \lambda)=D(x, \lambda)\left(D^{-1} \widetilde{V}_{0}^{-1} T_{0}^{-1} Z T_{0}\right)(t, \lambda) .
\end{aligned}
$$

Now we can define $\Gamma(x)$.

We let $\gamma_{i j}=+\infty$ as $(i, j)=(3,2),(4,2),(4,3)$ and $\gamma_{i j}=0$ for other $(i, j)$. The definition (22) of the matrix $D$ implies easily that under such choice all the exponential factors in (31) are bounded.

We introduce the Banach space $\mathbf{Z}$ of matrix functions $F(x)=\left(f_{i j}(x)\right)_{i, j=1}^{4}$ such that $f_{i j}$ are measurable on $(0,+\infty)$ and

$$
\|F(x)\|_{\mathbf{Z}}=\sup _{x>0}\|F(x)\|<\infty
$$

where

$$
\|F\|=\sqrt{\sum_{1 \leqslant i, j \leqslant 4}\left|f_{i j}\right|^{2}} .
$$

It is clear that $\widetilde{V}_{0} \in \mathbf{Z}$ for all $\lambda>0$. Let us show that $A(\lambda)$ is a contraction operator $\mathbf{Z}$ for sufficiently large $\lambda>0$. In order to do it, we shall need an estimate for the norm of the matrix $G=T_{0}^{-1} Z T_{0}$. We have (see (16), 20), 29)

$$
G=\left(I_{4}+X_{1}\right)^{-1} T_{0}^{-1}\left(B_{1} X-X^{\prime}\right) T_{0}+T_{0}^{-1} B_{2} T_{0}\left(I_{4}+X_{1}\right)-T_{0}^{-1} Z_{2} T_{0},
$$

where

$$
X_{1}=T_{0}^{-1} X T_{0}
$$

Lemma 1. For large $\lambda>0$

$$
\left\|X_{1}\right\|_{\mathbf{z}}=O\left(a_{\lambda}^{-1}+\lambda^{-3 / 4}+\lambda^{-(2+\alpha-\beta) / 3 \alpha}\right) .
$$

Proof. By simple calculations and (10)-(14) we have

$$
\left\|X_{1}(x, \lambda)\right\|=O\left[\left(\left|\nu_{2}\right|^{-\sigma / 2}\left|p_{1}^{\prime}\right| D^{-1 / 2}\right)(x, \lambda)\right], \lambda \gg 1,
$$

uniformly in $x \geqslant 0$. Inequalities (4), (5) sohw that for all $t \in(-\delta, \delta)$, where $\delta>0$ is independent of $\lambda$,

$$
\begin{aligned}
& \left|\nu_{2}\left(a_{\lambda}(1+t), \lambda\right)\right| \geqslant c_{1} \lambda^{(\alpha-\beta) / \alpha}|t|, \\
& \left|Q_{2}\left(a_{\lambda}(1+t), \lambda\right)\right| \leqslant c_{2} \lambda^{(\alpha+2-\beta) / 2 \alpha}|t|^{\frac{3}{2}},
\end{aligned}
$$

where $c_{1}, c_{2}$ are constants independent of $\lambda$. Then the functions $\delta_{1}(\lambda), \delta_{2}(\lambda)$ defined by (24) satisfy the estimates

$$
\left|\delta_{i}\right| \geqslant c_{i}^{-1} \lambda^{-(\alpha+2-\beta) / 3 \alpha} .
$$

By (33) it implies that

$$
\left(\nu_{2}^{-\sigma / 2} p_{1}^{\prime} D^{-1 / 2}\right)(x, \lambda)=O\left(a_{\lambda}^{-1}+\lambda^{-(\alpha+2-\beta) / 3 \alpha}\right)
$$

for all $x \in\left(a_{\lambda}(1-\delta), a_{\lambda}(1+\delta)\right)$. As $x \notin\left(a_{\lambda}(1-\delta), a_{\lambda}(1+\delta)\right)$, we employ again inequality (4), (5) to obtain

$$
\left(\nu_{2}^{-1 / 2} p_{1}^{\prime} D^{-1 / 2}\right)(x, \lambda)=O\left(\lambda^{-(\alpha+2-\beta) / 2 \alpha}+\lambda^{-3 / 4}+\lambda^{-(1 / 4+1 / 2 \beta)}\right) .
$$

The proof is complete. 
It follows from Lemma 1 that

where

$$
\|G\|=O\left(\left\|G_{0}\right\|\right)
$$

$$
G_{0}=T_{0}^{-1}\left(B_{1} X-X^{\prime}+B_{2}-Z_{2}\right) T_{0} .
$$

Let

$$
g(t, \lambda)=\frac{\left(p_{1}^{\prime}\right)^{2}+\left|q_{1}^{\prime}\right|}{D}+\frac{\left|p_{1}^{\prime \prime}\right|}{\sqrt{D}}+|\chi|\left(|p|+\lambda^{-1 / 2}|q|\right) .
$$

Lemma 2. For large $\lambda>0$, the estimate

$$
\left\|G_{0}(x, \lambda)\right\|=O\left(\left|\nu_{2}(x, \lambda)\right|^{-\frac{1}{2}}(|K(x, \lambda)|+g(x, \lambda))\right)
$$

holds true uniformly in $x \geqslant 0$.

Proof. Let

$$
G_{0}=\left(\begin{array}{ll}
G_{11} & G_{12} \\
G_{21} & G_{22}
\end{array}\right)
$$

where $G_{i j}$ are square matrices of second order. By relations (10)-(14), (16) and (29) we have

$$
\begin{aligned}
& G_{22}=\left(\begin{array}{cc}
0 & g_{1} \\
g_{2} & 0
\end{array}\right), \\
& g_{1}=-\frac{1}{8}\left|\nu_{2}\right|^{\sigma / 2} D^{-1 / 2}\left(\nu_{1}^{\prime} D^{\prime} D^{-3 / 2}+8 \chi p\right), \\
& g_{2}=\left|\nu_{2}\right|^{-\sigma / 2}\left(b_{4}^{2}+b_{4}^{\prime}-K+\frac{\nu_{2}^{\prime}\left(\nu_{2} \nu_{1}^{\prime}-\nu_{1} \nu_{2}^{\prime}\right)}{8 D^{3 / 2}}-\frac{\chi q}{2 \sqrt{D}}\right),
\end{aligned}
$$

where

$$
K(t, \lambda)=-\frac{5}{36} \frac{\left|\nu_{2}\right|}{Q_{2}^{2}}+\frac{5}{16} \frac{\left(q_{1}^{\prime}\right)^{2}}{\left(q_{1}-\lambda\right)^{2}}-\frac{1}{4} \frac{q_{1}^{\prime \prime}}{\left(q_{1}-\lambda\right)}-\frac{1}{8} \frac{q_{1}^{\prime} \nu_{1}^{\prime}}{\left(q_{1}-\lambda\right) \nu_{1}}+\frac{1}{4} \frac{\nu_{1}^{\prime \prime}}{\nu^{-1}}-\frac{3}{16} \frac{\left(\nu_{1}^{\prime}\right)^{2}}{\nu_{1}^{2}} .
$$

Now we see that $G_{22}$ satisfies estimate $(36)$. Similar calculations show that

$$
\left\|G_{11}\right\|=O\left(\nu_{1}^{-1 / 2} g(t, \lambda)\right), \quad\left\|G_{12}\right\|+\left\|G_{12}\right\|=O\left(\left|q_{1}-\lambda\right|^{-\sigma / 4} g(t, \lambda)\right) .
$$

The proof is complete.

Lemma 3. Under the assumptions of Theorem 1, the operator $A(\lambda)$ is bounded and its norm $\|A(\lambda)\|_{*}$ can be estimated as

$$
\begin{aligned}
& \|A(\lambda)\|_{*}=O\left(\lambda^{-m}\right), \quad \lambda \rightarrow+\infty, \\
& m=\min \left\{\frac{1}{4}, \frac{2+\alpha-\beta}{2 \alpha}\right\} .
\end{aligned}
$$

If in addition we assume the existence of the derivative $p$ absolutely continuous on the half-line $x \geqslant 0$, then

$$
m=\min \left\{\frac{3}{4}, \frac{1}{4}+\frac{1}{2 \beta}, \frac{2+\alpha-\beta}{2 \alpha}\right\} .
$$

Proof. By the choice of $\Gamma(x)$, all the exponentials factors in the kernel of the operator $A(\lambda)$ (see (31)) are bounded, therefore, (see (34), (36))

$$
\|A(\lambda)\|_{*}=O\left(\int_{0}^{\infty}\left|\nu_{2}\right|^{-1 / 2}(|K(t, \lambda)|+g(t, \lambda)) d t\right), \lambda \rightarrow+\infty .
$$

Then, arguing as in the proof of Lemma 1 in [10] and employing expression (39), inequalities (4), (6) and Condition 3), we obtain the desired estimate for $\|A(\lambda)\|$.

If the function $p$ has an absolutely continuous derivative, then at each place, where the function $p_{1}$ appears, it can be replaced by the function $p$ since the term $|X p|$ disappears in 
expression (35). This implies easily the second statement of the theorem. The proof is complete.

3.4. Proof of Theorem 1. It follows from Lemma 3 that the FSS of system (7) satisfies the asymptotic representation

$$
Y(x, \lambda)=T T_{0}\left(I_{4}+X_{1}\right)\left(\widetilde{V}_{0}+A_{\lambda} \widetilde{V}_{0}+O\left(\left\|A_{\lambda}\right\|_{*}^{2}\right)\right) D(x, \lambda),
$$

where $T, T_{0}, D$ are defined by (9), (22), (24), and $\widetilde{V}_{0}$ satisfies relation (26), (27). Hence, we concluce that the deficiency indices of the operator $L_{0}$ are equal to (2.2) and the equation for the eigenvalues of the operator $L$ is of the form:

$$
\operatorname{det}\left(C_{0} Y(0, \lambda) C_{1}^{T}\right)=0
$$

where

$$
C_{0}=\left(\begin{array}{cccc}
1 & 0 & 0 & 0 \\
0 & 0 & 1 & 0
\end{array}\right), \quad C_{1}=\left(\begin{array}{llll}
0 & 1 & 0 & 0 \\
0 & 0 & 1 & 0
\end{array}\right)
$$

Since $X_{1}(0, \lambda)=0, \nu_{1,2}(0, \lambda)= \pm \sqrt{\lambda}$, then

$$
C_{0} Y(0, \lambda) C_{1}^{T}=\lambda^{-3 / 8} \operatorname{diag}(1, \sqrt{\lambda}) C_{2} \widetilde{V}(0, \lambda)\left(I_{4}+A_{1}(\lambda) \widetilde{V}\right)(0, \lambda)+O\left(\left\|A_{\lambda}\right\|_{*}^{2}\right) C_{1}^{T},
$$

where

$$
C_{2}=\left(\begin{array}{cccc}
1 & 1 & 0 & 0 \\
0 & 0 & 1 & 0
\end{array}\right)
$$

Since

$$
\left(A_{1}(\lambda) \widetilde{V}_{0}\right)(0, \lambda) C_{1}^{T}=\left(A_{1}(\lambda) \widetilde{V}_{0} C_{1}^{T}\right)(0, \lambda),
$$

by the definition of $A_{1}(\lambda)$ (see (31)) we get

$$
\left(A_{1}(\lambda) \widetilde{V}_{0} C_{1}^{T}\right)(0, \lambda)=\left(\begin{array}{cc}
0 & 0 \\
0 & 0 \\
\alpha_{31} & 0 \\
\alpha_{41} & \alpha_{42}
\end{array}\right)
$$

and

$$
\alpha_{i j}=O\left(\int_{0}^{\infty}\left|\nu_{2}\right|^{-1 / 2}(|K(t, \lambda)|+g(t, \lambda)) \exp \left(-\delta Q_{1}\right) d t\right), \quad \lambda \rightarrow+\infty,
$$

for all $(i, j)=(3,1)$ and $(4,1)$. Here $\delta>0$ is a constant independent of $\lambda$. Taking into consideration that as $t<x_{0}$,

$$
|K(t, \lambda)|+g(t, \lambda)=\frac{5}{36}\left|\nu_{2}\right| Q_{2}^{-2}(t, \lambda)+|\chi|\left(|p|+\lambda^{-1 / 2}|q|\right),
$$

we obtain

$$
\alpha_{i j}=O\left(\lambda^{-1 / 4} \int_{0}^{x_{0}+1}|\chi p| \exp \left(-\delta_{0} Q_{1}\right) d t\right)+O\left(\lambda^{-3 / 4}\right)+O\left(Q_{2}^{-2}(0, \lambda)\right), \quad(i, j) \neq(4,2),
$$

where $\delta_{0}$ is a positive constant. In order to calculate $\alpha_{42}$, we note that (see (27))

$$
\operatorname{det}\left(\widetilde{V_{02}}(x, \lambda)\right)=\operatorname{det}\left(\widetilde{V_{02}}(\infty, \lambda)\right)=1,
$$

therefore,

$$
{\widetilde{V_{02}}}^{-1}=\left(\begin{array}{cc}
\omega_{22} & -\omega_{12} \\
-\omega_{21} & \omega_{11}
\end{array}\right)
$$


where $\omega_{i j}$ are the entries of the matrix $\widetilde{V}_{02}$. Then (see $(37),(38)$ )

$$
\begin{aligned}
& \alpha_{42}=\int_{0}^{\infty}\left(g_{1} \omega_{12} \omega_{21}-g_{2} \omega_{11}^{2}\right) \exp \left(-2 d(t, \lambda) Q_{1}(t, \lambda)\right) d t+O(\alpha(\lambda)), \\
& \alpha(\lambda)=\int_{0}^{\infty}\left\|G-G_{0}\right\| \exp \left(-2 d(t, \lambda) Q_{2}(t, \lambda)\right) d t
\end{aligned}
$$

The straightforward calculations give

$$
\alpha(\lambda)=O\left(\int_{0}^{\infty}\left|q_{1}-\lambda\right|^{-1 / 2}\left(\left(\left(p_{1}^{\prime}\right)^{2}+\left|q_{1}^{\prime \prime}\right|\right) D^{-1 / 2}+\left|p_{1}^{\prime \prime}\right|\right) p_{1}^{\prime} D^{-1 / 2} d t\right) .
$$

Employing inequalities (4), (6), Condition 3) and the sign-definiteness of the second derivatives of $p$ and $q$, by simple calculations we obtain

$$
\alpha_{42}(\lambda)=\frac{1}{2} \int_{0}^{a_{\lambda}}\left(g_{1}-g_{2}\right) d t+O(b(\lambda))+O\left(\lambda^{-m_{1}}\right)
$$

where $b(\lambda)$ is defined by formula $(19)$,

$$
m_{1}=\min \left\{\frac{2+\alpha-\beta}{3 \alpha}+\frac{1}{\alpha}, \frac{3}{2}, \frac{1}{2}+\frac{2}{\alpha}\right\} .
$$

Then, taking into consideration (42), by (40), (41) we get

$$
\lambda^{-1 / 8} \operatorname{det}\left(C_{0} Y(0, \lambda) C_{1}^{T}\right)=\omega_{11}(0, \lambda)+\alpha_{42}(\lambda) \omega_{12}(0, \lambda)+O\left(\beta(\lambda)+\left\|A_{\lambda}\right\|_{*}^{2}+Q_{2}^{-2}(0, \lambda)\right) .
$$

Replacing in the latter expression $\omega_{11}(0, \lambda)$ and $\omega_{12}(0, \lambda)$ by their asymptotics in accordance with (27), we obtain (17). The proof is complete.

\section{AsYmptotics OF THE SPECTRUM}

In this section we obtain the asymptotics for the eigenvalues of the operator $L$, when $p$ and $q$ have the form

$$
p(x)=x^{\beta}, \quad q(x)=x^{\alpha}, \quad 0<\frac{\alpha}{2}<\beta<\alpha+2 .
$$

We shall show that the leading term in the asymptotic series for $\lambda_{k}$ depends on the value of $\operatorname{sgn}(\beta-2)$.

4.1. First approximation for the solution to equation (17). It follows from equation (17) that

$$
\Phi\left(\lambda_{k}\right)=\nu_{k} \pi+o(1), \quad k \rightarrow \infty
$$

for each fixed pair $(\alpha, \beta)$ satisfying (43). We shall show below (Lemma 6) that $\nu_{k}=k$.

Lemma 4. The asymptotics of the spectrum of the operator $L$ in the case $p(x)=x, q(x)=x^{2}$ is of the form

$$
\lambda_{k}=\left[\frac{3}{2} \pi\left(k-\frac{1}{4}\right)\right]^{4 / 3}-\frac{1}{16}\left[3 / 2 \pi\left(k-\frac{1}{4}\right)\right]^{-2 / 3}+O\left(k^{-1}\right) .
$$

Proof. It is easy to see that under the assumptions of the lemma, $L=L_{1}^{2}$, where $L_{1}$ is the Sturm-Liouville operator generated by the expression $-y^{\prime \prime}+x y$ and the boundary condition $y(0)=0$ in $L(0,+\infty)$. The asymptotics of the eigenvalues of $L_{1}$ is known [11, Lm. 5]. It implies the statement of the lemma. 
Under the assumptions of Lemma 4 .

$$
\lim _{x \rightarrow \infty} \frac{\mu_{i}(x, \lambda)}{\mu_{i+2}(x, \lambda)}=1, \quad i=1,2,
$$

that is, we deal with the case of the asymptotically multiple roots [3, Ch. V, Sect. 4]. In this case $D(x, \lambda)=\lambda$ and the integral

$$
\int_{0}^{\infty}\left|\nu_{2}\right|^{-1 / 2}(|K(t, \lambda)|+g(t, \lambda)) d t
$$

diverges. Nevertheless, up to some minor changes, Theorem 1 can be extended to the case $q(x)=p^{2}(x)$.

Lemma 5. Let $q(x)=x^{\alpha}, p(x)=x^{\beta}$, where $(\alpha, \beta) \in \Omega$,

$$
\Omega=\{(\alpha, \beta): 0<\alpha / 2<\beta<\alpha+2 \text { or } 0<\alpha / 2=\beta<2\} .
$$

Then

$$
\sin \Phi(\lambda)=o(1), \lambda \rightarrow \infty,
$$

uniformly over each compact set $K \subset \Omega$.

Proof. Let $b_{\lambda}=(1+\delta) a_{\lambda}, \delta>0$ be independent of $\lambda$. Under the mentioned $\alpha, \beta$, Theorem 7 remains true if in the definition of the space $\mathbf{Z}$ and operator $A(\lambda)$ the half-line $[0, \infty)$ is replaced by the segment $\left[0, b_{\lambda}\right]$. Therefore, the FSS of system (7) has the asymptotics

$$
Y=T T_{0} \widetilde{V}_{0}\left(I_{4}+o(1)\right) D(x, \lambda), \quad \lambda \rightarrow \infty,
$$

uniformly in $x \in\left[0, b_{\lambda}\right]$.

By the WKB-method one can show easily that as $0<\alpha<4$, relation (45) is true on the half-line $\left[b_{\lambda}, \infty\right)$. It implies (44). The proof is complete.

Lemma 6. Let $p$ and $q$ are of form (43). Then

$$
\Phi\left(\lambda_{k}\right)=k \pi+o(1), \quad k \rightarrow \infty .
$$

Proof. By Lemma 5 we have

$$
\Phi\left(\lambda_{k}\right)=\nu_{k} \pi+o(1), \quad k \rightarrow \infty .
$$

But $\lambda_{k}=\lambda_{k}(\alpha, \beta)$ is continuous on $\Omega$ [20, Example 1]. Therefore, $\nu_{k}(\alpha, \beta)$ is also continuous on $\Omega$. Since $\nu_{k}(2,1)=k$ (Lemma 9), then $\nu_{k}(\alpha, \beta)=k$ on $\Omega$. The proof is complete.

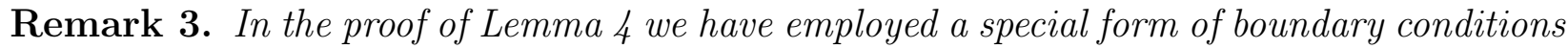
(2.2). In the case of arbitrary self-adjoint boundary conditions one can proceed as in the proof of Lemma 5 in [11] observing that as $p(x)=x, q(x)=x^{2}$, the substitution

$$
\begin{gathered}
Y=T U, \\
T=\left(\begin{array}{cc}
1 & 1 \\
1 & -1
\end{array}\right), \quad Y=\left(y_{1}, y_{2}\right)^{T}, \quad y_{1}=\sqrt{\lambda} y, \quad y_{2}=-y^{\prime \prime}+x y,
\end{gathered}
$$

transforms the equation $\mathcal{L}(y)=\lambda y$ to the system $-V^{\prime \prime}+x V=\sqrt{\lambda} \operatorname{diag}(1,-1) V$, whose solutions are expressed directly in terms of the Airy functions. 


\subsection{Asymptotics of the spectrum.}

Theorem 2. Assume that the functions $p$ and $q$ are of form (2.40). Then as $1<\beta<2$, the eigenvalues of the operator $L$ have the asymptotics

$$
\lambda_{k}=m_{k}^{\frac{2 \alpha}{2+\alpha-\beta}}-\frac{2 \alpha}{2+\alpha-\beta} C_{0}\left\{C_{1} m_{k}^{\frac{2 \alpha}{2+\alpha-\beta}-\frac{(2 \beta-\alpha)(2-\beta)}{2 \beta(2+\alpha-\beta)}}+C_{2} m_{k}^{-\frac{(\alpha+2 \beta)(2-\beta)}{2 \beta(2+\alpha-\beta)}}\right\}+O\left(k^{-\frac{4(\beta-\alpha)}{2+\alpha-\beta}}\right),
$$

where

$$
\begin{aligned}
& m_{k}=C_{0} \pi\left(k-\frac{1}{4}\right), \quad C_{0}=\frac{\sqrt{2} \alpha \Gamma\left(\frac{3}{2}+\frac{2-\beta}{2 \alpha}\right)}{\Gamma\left(\frac{3}{2}\right) \Gamma\left(\frac{-\beta+2}{2 \alpha}\right)} \\
& C_{1}=\frac{1}{\sqrt{2}} \int_{0}^{\infty} t^{-\beta / 2}\left(t^{\beta}+\sqrt{t^{2 \beta}+1}\right)^{-3 / 2}\left(\sqrt{2} t^{\beta / 2}+\left(t^{\beta}+\sqrt{t^{2 \beta}+1}\right)^{1 / 2}\right)^{-1} d t \\
& C_{2}=\frac{\beta}{8} \int_{0}^{1} t^{-1 / \beta}(1-t)^{-5 / 4+1 / 2 \beta}(1+t)^{-3 / 4+1 / 2 \beta}\left((\beta-1) / \beta+3 t / 4-2 t^{3}+t^{4}\right) d t .
\end{aligned}
$$

As $0<\beta \leqslant 1$, similar formulae hold true.

In order to obtain the asymptotics for $\lambda_{k}$ by (46) and (17), we need to study the behavior of the functions $Q_{2}(0, \lambda), K(\lambda), \beta(\lambda)$ for large $\lambda>0$, where $p$ and $q$ are of form (43).

Lemma 7. If $0<\beta<2$, then

$$
-Q_{2}(0, \lambda)=\lambda^{(2+\alpha-\beta) / 2 \alpha}\left(C_{0}^{-1}+\sum_{k=1}^{n-1} a_{k} \lambda^{-(2 \beta-\alpha) k / \alpha}\right)+O\left(\lambda^{-(2 \beta-\alpha) n / \alpha} \rho(\lambda)\right),
$$

as $\lambda \rightarrow+\infty$, where $n=n(\beta) \in \mathbb{N}$ are defined by inequalities (48), $a_{k}=f_{k} I_{k}, f_{k}$ and $I_{k}$ are determined by the formulae 47) and (50),

$$
\rho(\lambda)= \begin{cases}\ln \lambda, & \beta=2 /(4 n+1), \\ \lambda^{-\{1 / 4-1 / 2 \beta\}}, & \text { for other } \beta,\end{cases}
$$

$\{x\}$ denotes the fractional part of a number $x$.

Proof. The substitution $x=a_{\lambda} t$ transforms $Q_{2}(0, \lambda)$ to the form

$$
-Q_{2}(0, \lambda)=\lambda^{(2+\alpha-\beta) / 2 \alpha} \int_{0}^{1}\left(1-t^{\alpha}\right)^{1 / 2}\left[t^{\beta}+\left(t^{2 \beta}+\varepsilon\left(1-t^{\alpha}\right)\right)^{1 / 2}\right]^{-1 / 2} d t \equiv \lambda^{(2+\alpha-\beta) / 2 \alpha} I(\varepsilon) .
$$

where $\varepsilon=\lambda^{-(2 \beta-\alpha) / \alpha}$.

Since the function

$$
f(z)=\left(1+(1+z)^{1 / 2}\right)^{-1 / 2}
$$

in analytic in the unit circle, then

$$
f(z)=\sum_{k=1}^{\infty} f_{k} z^{k}, \quad|z|<1
$$

and $f_{0}=1 / \sqrt{2}$. Let $n$ be a natural number satisfying the inequalities

$$
\frac{1}{2 \beta}-\frac{1}{4} \leqslant n<\frac{1}{2 \beta}+\frac{3}{4} .
$$

We let

$$
R_{n}(\varepsilon)=I(\varepsilon)-\sum_{k=0}^{n-1} f_{k} I_{k} \varepsilon^{k}
$$

where

$$
I_{k}=\int_{0}^{1}\left(1-x^{\alpha}\right)^{k+1 / 2} x^{-(4 k+1) \beta / 2} d x
$$


We have [22, Ch. XII, No. 855.42]

$$
I_{k}=\frac{\Gamma\left(\frac{2 k+3}{2}\right) \Gamma\left(\frac{-(4 k+1) \beta+2}{2 \alpha}\right)}{\alpha \Gamma\left(\frac{2 k+3}{2}+\frac{2-(4 k+1) \beta}{2 \alpha}\right)} .
$$

Since

$$
\left[t^{\beta}+\sqrt{t^{2 \beta}+\varepsilon\left(1-t^{\alpha}\right)}\right]^{-1 / 2}=t^{-\beta / 2} f\left(\varepsilon\left(1-t^{\alpha}\right) / t^{2 \beta}\right),
$$

by the uniform convergence of series $(47)$ in the circle $|z| \leqslant r<1$, the function

$$
r_{n}(t, \varepsilon)=\left[t^{\beta}+\sqrt{t^{2 \beta}+\varepsilon\left(1-t^{\alpha}\right)}\right]^{-1 / 2}-\sum_{k=0}^{n-1} f_{k} \cdot\left(1-t^{\alpha}\right)^{k} t^{-(2 k+1 / 2) \beta} \varepsilon^{k}
$$

satisfies the estimate

$$
\left|r_{n}(t, \varepsilon)\right| \leqslant C_{n} \varepsilon^{n} t^{-(2 n+1 / 2) \beta}, \quad t \in\left[(1+\delta) \varepsilon^{1 / 2 \beta}, 1\right],
$$

where $M, \delta$ are positive constants independent of $\varepsilon$. Then, since as $t \in\left(0,(1+\delta) \varepsilon^{1 / 2 \beta}\right)$,

$$
\left|r_{n}(t, \varepsilon)\right| \leqslant M_{n}^{\prime} \varepsilon^{n-1} t^{-(2 n-3 / 2) \beta},
$$

where $M^{\prime}>0$ is independent of $t$ and $\varepsilon$, then

$$
R_{n}(\varepsilon) \leqslant M \varepsilon^{n} \int_{(1+\delta) \varepsilon^{1 / 2 \beta}}^{1} t^{-(2 n+1 / 2) \beta} d t+M^{\prime} \varepsilon^{n-1} \int_{0}^{(1+\delta) \varepsilon^{1 / 2 \beta}} t^{-(2 n-3 / 2) \beta} d t .
$$

According to (48),

$$
-2 \beta<-(2 n+1 / 2) \beta+1 \leqslant 0, \quad 0<-(2 n-3 / 2) \beta \leqslant 2 \beta,
$$

so that

$$
R_{n}(\varepsilon)=\left\{\begin{array}{ll}
O\left(\varepsilon^{n} \ln \varepsilon\right), & \beta=2 /(4 n+1), \\
O\left(\varepsilon^{n-\{1 / 4-1 / 2 \beta\}}\right), & 1 / 2 \beta-1 / 4 \notin \mathbb{N},
\end{array} \quad \varepsilon \rightarrow+0 .\right.
$$

By relations (49), (50) it implies the statement of the lemma.

Lemma 8. As $1<\beta<2$ and $\lambda \rightarrow+\infty$, the estimate

$$
K(\lambda)=C_{2} \lambda^{-(\beta+2) / 4 \beta}+O\left(\lambda^{-(2+\alpha-\beta) / 2 \alpha}+\lambda^{-3 / 4}\right)
$$

holds true.

Proof. Since $\beta>1$, then $p^{\prime}$ is absolutely continuous on $[0,+\infty)$ and this is why in expressions (18) and (12) we can take $p$ and $q$ instead of $p_{1}$ and $q_{1}$, respectively, and $\chi \equiv 0$. Hence, by (18) and (12) we obtain

$$
\begin{aligned}
& K(\lambda)=K_{1}(\lambda)+K_{2}(\lambda)+O\left(\lambda^{-3 / 4}\right), \\
& K_{1}(\lambda)=-\frac{1}{2} \int_{0}^{a_{\lambda}} \nu_{2}^{-1 / 2} K(t, \lambda) d t \\
& K_{2}(\lambda)=\frac{1}{16} \int_{0}^{a_{\lambda}} \nu_{2}^{-1 / 2}\left(\frac{2 p^{\prime 2}}{D}+4\left(\frac{p^{\prime}}{\sqrt{D}}\right)^{\prime}+\frac{\nu_{2}^{\prime}\left(\nu_{2} \nu_{1}^{\prime}-\nu_{1} \nu_{2}^{\prime}\right)}{8 D^{3 / 2}}\right) d t .
\end{aligned}
$$

By straightforward calculations we confirm that (see (39p)

$$
\begin{aligned}
K_{1}(\lambda) & =-\frac{1}{8} \int_{0}^{a_{\lambda}} \nu_{2}^{-1 / 2} \frac{\nu_{1}^{\prime \prime}}{\nu_{1}} d t+\frac{3}{32} \int_{0}^{a_{\lambda}} \nu_{2}^{-1 / 2}\left(\frac{\nu_{1}^{\prime}}{\nu_{1}}\right)^{2} d t+O\left(\lambda^{-(2+\alpha-\beta) / 2 \alpha}\right) \\
& =K_{11}(\lambda)+K_{12}(\lambda)+O\left(\lambda^{-(2+\alpha-\beta) / 2 \alpha}\right) .
\end{aligned}
$$


The integrals $K_{11}(\lambda), K_{12}(\lambda), K_{2}(\lambda)$ are of the same nature and one can find easily their asymptotics. We have

$$
\begin{aligned}
& K_{11}(\lambda)=-\frac{\beta}{8} \lambda^{-1 / 2} \int_{0}^{a_{\lambda}} k(t, \lambda) d t+O\left(\lambda^{-(2+\alpha-\beta) / 2 \alpha}\right), \\
& k(t, \lambda)=t^{\beta / 2}\left(t^{\beta}+\sqrt{t^{2 \beta}+\lambda}\right)^{-1 / 2}\left[\beta-1+(2 \beta-1) t^{\beta}\left(t^{2 \beta}+\lambda\right)^{-1 / 2}-\beta t^{2 \beta}\left(t^{2 \beta}+\lambda\right)^{-1}\right] .
\end{aligned}
$$

Making the change of the variables $t \longmapsto s=\left(1+t^{-2 \beta}\right)^{-1 / 2}$, we obtain

$K_{11}(\lambda)=-\frac{\beta}{8} \lambda^{-(\beta+2) / 4 \beta} \int_{0}^{a_{\lambda}} s^{-1 / \beta}(1-s)^{-(5 \beta+2) / 4 \beta}((\beta-1) / \beta+s(1-s)) d s+O\left(\lambda^{-(2+\alpha-\beta) / 2 \alpha}\right)$.

Making similar calculations for $K_{12}(\lambda), K_{2}(\lambda)$ as $1<\beta<2$, we obtain (51). The proof is complete.

Completion of the proof of Theorem 2. According to (19),

$$
b(\lambda)=b_{1}(\lambda)+\exp \left(i \int_{0}^{a_{\lambda}}\left|\nu_{2}\right|^{1 / 2} d t\right) b_{2}(\lambda)
$$

where

$$
\begin{aligned}
& b_{1}(\lambda)=\int_{0}^{a_{\lambda} / 2} \exp \left(i \int_{0}^{t}\left|\nu_{2}\right|^{1 / 2} d t\right) B(t, \lambda) d t, \\
& b_{2}(\lambda)=\int_{a_{\lambda} / 2}^{a_{\lambda}} \exp \left(-i \int_{t}^{a_{\lambda}}\left|\nu_{2}\right|^{1 / 2} d t\right) B(t, \lambda) d t, \\
& B(t, \lambda)=\left|\nu_{2}\right|^{-1 / 2}\left[\left(\frac{p^{\prime}}{\sqrt{D}}\right)^{2}+\frac{p^{\prime \prime}+\left|q_{1}^{\prime \prime}\right|}{\sqrt{D}}\right] .
\end{aligned}
$$

Integrating by parts, we have

$$
b_{1}(\lambda)=O\left(\lambda^{-\delta_{1}}\right), \quad \lambda \rightarrow+\infty
$$

where

$$
\delta_{1}=\min \left\{\frac{\alpha+2-\beta}{2 \alpha}, \frac{4-\beta}{4 \beta}, \frac{4+2 \beta-\alpha}{2 \alpha}\right\} .
$$

Let us estimate $b_{2}$. We let $Q(x, \lambda)=\int_{t}^{a_{\lambda}}\left|\nu_{2}\right|^{1 / 2} d t$. Since

$$
Q(x, \lambda)=-3 / 2 \int_{t}^{a_{\lambda}}\left(q^{\prime}\right)^{-1} \nu_{1}^{-1 / 2} d\left((\lambda-q)^{3 / 2}\right),
$$

and the functions $q^{\prime}$ and $\nu_{1}$ are monotonous, then

$$
A_{1} a_{\lambda}^{1-\alpha-\beta / 2} \leqslant \frac{Q(x, \lambda)}{(\lambda-q(x))^{3 / 2}} \leqslant A_{2} a_{\lambda}^{1-\alpha-\beta / 2}, \quad x \in\left[a_{\lambda} / 2, a_{\lambda}\right], \quad \lambda \gg 1,
$$

where $A_{1}, A_{2}$ are positive constants independent of $\lambda$. For each $k=0,1, \ldots$

$$
\frac{\partial^{k}}{\partial x^{k}}\left[\left(\frac{\left(p^{\prime}\right)^{2}+q_{1}^{\prime \prime}}{\sqrt{D}}\right)^{2}+\frac{p^{\prime \prime}}{\sqrt{D}}\right]\left(x, a_{\lambda}\right)=O\left(x^{-2-k}\right), x \geqslant a_{\lambda} / 2,
$$

uniformly in $\lambda \geqslant \Lambda_{0} \gg 1$. Then

$$
(\lambda-q(t))^{-1} B(t, \lambda)=Q(t, \lambda)^{-2 / 3} \lambda^{-\gamma} \psi(t, \lambda), \quad x \in\left[a_{\lambda} / 2, a_{\lambda}\right], \quad \lambda \gg 1,
$$

where $\gamma=2(\alpha+2-\beta) / 3 \alpha$, the function $\psi$ and its derivatives in $x$ satisfy the estimates

$$
\psi^{(k)}(x, \lambda)=O\left(x^{-k}\right), \quad x \geqslant a_{\lambda} / 2,
$$


uniformly in $\lambda \geqslant \Lambda_{0} \gg 1$. Making the change $x \mapsto Q=Q(x, \lambda)$ in the integral $g_{2}$, we obtain

$$
b_{2}(\lambda)=\lambda^{-\gamma} \int_{0}^{A(\lambda)} e^{-i Q} Q^{-2 / 3} \Psi(Q, \lambda) d Q
$$

where $A(\lambda)=Q\left(a_{\lambda} / 2, \lambda\right)$, and by (53) the function $\Psi(Q, \lambda)=\psi(t(Q), \lambda)$ satisfies the estimates

$$
\left|\frac{\partial^{k}}{\partial Q^{k}} \Psi(Q(t, \lambda), \lambda)\right| \leqslant \frac{B_{k}}{\left|\nu_{2}(t, \lambda)\right|^{k / 2} a_{\lambda}^{k}}, \quad t \in\left[a_{\lambda} / 2, a_{\lambda}\right], \quad \lambda \gg 1,
$$

$B_{k}>0, k \in \mathbb{N}$, are independent of $t, \lambda$. It follows that

$$
b_{21}(\lambda):=\int_{0}^{1} e^{-i Q} Q^{-2 / 3}\left(\lambda^{-\gamma} \Psi(Q, \lambda)\right) d Q=O\left(\lambda^{-\gamma}\right), \quad \lambda \rightarrow+\infty .
$$

By inequalities (52) we see that as $Q(t, \lambda) \geqslant 1,\left|\nu_{2}(t, \lambda) t\right| \geqslant C \lambda^{(\alpha+2-\beta) / 3 \alpha}$, where $C>0$ is independent of $t, \lambda$. By (54) it follows that for some $n \in \mathbb{N}$

$$
\int_{1}^{A(\lambda)} q^{-2 / 3}\left|\frac{\partial^{n}}{\partial Q^{n}} \Psi(Q(t, \lambda), \lambda)\right| d Q=O\left(\lambda^{-\gamma}\right), \quad \lambda \rightarrow+\infty, \quad i=1,2 .
$$

This is why, integrating by parts $n$ times in $b_{22}:=b_{2}-b_{21}$ and taking into consideration inequalities (54), we obtain

$$
\begin{aligned}
& b_{2}(\lambda)=O\left(\lambda^{-\gamma}\right), \lambda \rightarrow+\infty, \\
& b(\lambda)=O\left(\lambda^{-(2+\alpha-\beta) / 2 \alpha}+\lambda^{-1+\beta / 4}\right), \quad \lambda \rightarrow+\infty .
\end{aligned}
$$

Substituting the obtained expression for $K(\lambda), Q_{2}(0, \lambda), b(\lambda)$ into equation (17), solving it w.r.t $\lambda_{k}$ and taking into consideration (46), we arrive at the statement of the theorem.

Remark 4. In the case $0<\beta \leqslant 1$, we can not neglect the terms in formulae (18) and (12) involving the cut-off function $\chi$. It produces additional terms in formulae (51) and (55). Moreover, additional difficulties arise related to the non-integrability of the functions $p^{2}$ and $p^{\prime \prime}$ at zero and this is why the expansions like (49) for the integrals in the expression for $K(\lambda)$ depend on a particular value of $\beta$. This is exactly the reason why we have restricted ourselves by the case $1<\beta<2$ in Theorem 1 .

As $\beta \geqslant 2$, the asymptotics of the integrals $Q_{2}(0, \lambda), K(\lambda)$ can be studied in the same way as for $\beta<2$. As $\beta>2$, the formulae for the mentioned integrals are similar to the case $\beta<2$, a formal difference is due to the fact that

$$
\frac{\beta+2}{4 \beta}-\frac{2+\alpha-\beta}{2 \alpha}=\frac{(\beta-2)(2 \beta-\alpha)}{4 \alpha \beta}>0
$$

as $\beta>2$. In the case $\beta=2$, the formulae for $Q_{2}(0, \lambda)$ and $K(\lambda)$ has its own features. Omitting intermediate steps similar to the case $\beta<2$, we provide the final form of the asymptotic formulae as $\lambda \rightarrow+\infty$ :

$$
\begin{aligned}
& -Q_{2}(0, \lambda)= \begin{cases}\widetilde{C}_{1} \lambda^{(\beta+2) / 4 \beta}-\widetilde{C}_{2} \lambda^{(2+\alpha-\beta) / 2 \alpha}+O\left(\lambda^{(2 \alpha+2-3 \beta) / 4 \beta}\right), & \beta>2, \\
\widetilde{C}_{3} \lambda^{1 / 2} \ln \lambda+\widetilde{C}_{4} \lambda^{1 / 2}+O\left(\lambda^{(\alpha-2) / 4}\right)+O\left(\lambda^{(3 \alpha-8) / 2 \alpha}\right), & \beta=2,\end{cases} \\
& K(\lambda)= \begin{cases}O\left(\lambda^{(\alpha+2-\beta) / 2 \alpha}\right), & \beta>2, \\
O\left(\lambda^{-1 / 2} \ln \lambda\right), & \beta=2,\end{cases} \\
& b(\lambda)=o(K(\lambda)),
\end{aligned}
$$


where

$$
\begin{aligned}
& \widetilde{C}_{1}=\int_{0}^{\infty} \frac{d t}{\sqrt{t^{\beta}}+\sqrt{t^{2 \beta}+1}}, \quad \widetilde{C}_{2}=\frac{\sqrt{2}}{\beta-2}+\frac{1}{\sqrt{2}} \int_{0}^{1} \frac{t^{\alpha-\beta / 2} d t}{1+\sqrt{1-t^{\alpha}}}, \quad \widetilde{C}_{3}=\frac{4-\alpha}{4 \sqrt{2} \alpha}, \\
& \widetilde{C}_{4}=\int_{0}^{\infty}\left[\frac{1}{\sqrt{t^{2}+\sqrt{t^{4}+1}}}-\frac{1}{\sqrt{2}(t+1)}\right] d t-\frac{1}{\sqrt{2}} \int_{0}^{1} \frac{t^{\alpha-1} d t}{1+\sqrt{1-t^{\alpha}}} .
\end{aligned}
$$

Theorem 3. Assume that the functions $p$ and $q$ are of form (43). Then the spectrum of the operator $L$ has the asymptotics

a) as $\beta>2$,

$$
\begin{aligned}
& \lambda_{k}=m_{k}^{\frac{4 \beta}{2+\beta}}+\frac{4 \beta}{2+\beta} \frac{\widetilde{C}_{2}}{\widetilde{C}_{1}} m_{k}^{\frac{4 \beta \alpha-(\beta-2)(2 \beta-\alpha)}{(2+\beta) \alpha}}+O\left(k^{\delta}\right), \\
& \delta=\max \left\{\frac{(\beta-2)(2 \beta+\alpha)}{\alpha(\beta+2)}, \frac{2 \alpha}{\beta+2}\right\},
\end{aligned}
$$

b) as $\beta=2$,

$$
\lambda_{k}=\exp \left(-\frac{\widetilde{C}_{4}}{\widetilde{C}_{3}}\right) \Psi^{2}\left(\mu_{k}\right)\left[1+O\left(k^{-\frac{4-\alpha}{2}}(\ln k)^{-1+\frac{4-\alpha}{2}}+\left(\frac{k}{\ln k}\right)^{-\frac{8}{\alpha}+2}\right)\right],
$$

where

$$
m_{k}=\frac{\pi(4 k-1)}{4 \widetilde{C}_{1}}, \quad \mu_{k}=\frac{\pi(4 k-1) \exp \left(\widetilde{C}_{4} / 2 \widetilde{C}_{3}\right)}{8 \widetilde{C}_{3}},
$$

the constants $\widetilde{C}_{i}, i=\overline{1,4}$, are defined by (58), (59), $\Psi(\mu)$ is the inversion function for $\varphi(\mu)=$ $\mu \ln \mu$ satisfying asymptotic expansion (60) for large $\mu$.

Proof. Let $\beta=2$. In view of (56), (57), by equations (17), 46) we find

$$
\widetilde{C}_{3} \lambda_{k}^{1 / 2} \ln \lambda_{k}+\widetilde{C}_{4} \lambda_{k}^{1 / 2}+O\left(\lambda_{k}^{\alpha-2 / 4}+\lambda_{k}^{(3 \alpha-8) / 2 \alpha}\right)=\pi\left(k-\frac{1}{4}\right)
$$

which implies

$$
\lambda_{k}=\exp \left(-\frac{\widetilde{C}_{4}}{\widetilde{C}_{3}}\right) \Psi^{2}\left(\mu_{k}\left(1+O\left(\frac{\lambda_{k}^{(\alpha-4) / 4}}{\ln \lambda_{k}}+\frac{\lambda_{k}^{(\alpha-4) / \alpha}}{\ln \lambda_{k}}\right)\right)\right) .
$$

For large $\mu>0$, the function $\Psi(\mu)$ has asymptotic expansion [21, Ch. I, Sect. 5]:

$$
\Psi(\mu)=\frac{\mu}{\ln \mu}\left(1+\sum_{m=0, k=1} c_{k m}(\ln \ln \mu)^{k}(\ln \mu)^{-k-\mu}\right),
$$

where the coefficients $c_{k m}$ can be found explicitly. It implies Statement b).

Statement a) can be proved in the same way.

\section{BIBLIOGRAPHY}

1. M.A. Naimark. Linear differential operators. Fizmatlit, Moscow. 1969. [Frederick Ungar Publishing Co., N.Y. Part I. 1967; Part II. 1968.]

2. A.G. Kostyuchenko, I.S. Sargsyan. Distribution of eigenvalues. Selfadjoint ordinary differential operators. Nauka, Moscow (1979). (in Russian).

3. M.V. Fedoryuk. Asymptotic analysis: linear ordinary differential equations. Nauka, Moscow. 1983. [Springer-Verlag, Berlin. 1993.]

4. R.E. Langer. The asymptotic solutions of ordinary linear differential equations of the second order with special reference to the Stokes' phenomenon // Bull. Amer. Math. Soc. 40:8, 545-582 (1934). 
5. M.A. Evgrafov, M.V. Fedoryuk. Asymptotic behaviour as $\lambda \rightarrow \infty$ of the solution of the equation $w^{\prime \prime}(z)-p(z, \lambda) w(z)=0$ in the complex $z$-plane // Uspekhi Matem. Nauk. 21:1, 3-50 (1966). [Russ. Math. Surv. 21:1, 1-48 (1966).]

6. M. Giertz. On the solution in $L^{2}$ of $y^{\prime \prime}+(\lambda-q(x)) y=0$ when $q$ is rapidly increasing // Proc. London Math. Soc. 14:53, 53-73 (1964).

7. A.G. Alenitsyn. Asymptotic properties of the spectrum of a Sturm-Liouville operator in the case of a limit circle // Differ. Uravn. 12:3, 428-437 (1976). [Differ. Equat. 12:3, 298-305 (1977).]

8. V.A. Lyubishkin. The calculation of the regularized trace of the Sturm-Liouville operator in the case of the limit Weyl circle // Tr. Semin. Im. I. G. Petrovskogo. 6, 167-194 (1981). (in Russian).

9. F.V. Atkinson, G.T. Fulton. Asymptotic formulae for eigenvalues of limit circle problems on a half line // Ann. Math. Pure and Appl. 135:4, 368-398 (1983).

10. H.H. Murtazin, T.G. Amangil'din. The asymptotic expansion of the spectrum of a sturm-liouville operator. // Matem. sborn. 110(152):1, 135-149 (1979). [Math. USSR. Sb. 38:1, 127-141 (1981).]

11. Kh.K. Ishkin. Asymptotic behavior of the spectrum and the regularized trace of higher-order singular differential operators // Differ. Uravn. 31:10, 1658-1668 (1995). [Differ. Equat. 31:10, 16221632 (1995).]

12. W.N. Everitt. On the limit-point classification of fourth-order differential equations // J. London. Math. Soc. 44:1, 273-281 (1969).

13. M.S.P. Eastham. The limit-2 case of fourth-order differential equations // Quart. J. Math. 22:1, 131-134 (1971).

14. A. Devinatz. The deficiency index of certain fourth-order ordinary self-adjoint differential equations // Quart. J. Math. 23:4, 267-286 (1972).

15. A. Devinatz. On limit-2 fourth-order differential equations // J. Lond. Math. Soc. 7:1, 135-146 (1973).

16. W.D. Evans. On non-integrable square solutions of a fourth-order differential equations and the limit-2 classification// J. London. Math. Soc. 7:2, 343-354 (1973).

17. D. Hinton. Limit point criteria for differential equations // Canad. J. Math. 24:2, 293-305 (1972).

18. L.I. Anikeeva. Asymptotic behavior of the solution of the equation $y^{(4)}-a\left(x^{\beta} y^{\prime}\right)^{\prime}+b x^{\alpha} y=\lambda y$ as $x \rightarrow \infty / /$ Vestnik MGU. Ser I. 31:6, 44-52. [Mosc. Univ. Math. Bull. 31:5-6, 109-116 (1976).]

19. A. Erdélyi, W. Magnus, F. Oberhettinger, F.G. Tricomi. Higher transcendental functions. Vol. II. Bateman Manuscript Project. McGraw-Hill Book Co., New York (1953).

20. Kh.K. Ishkin. On continuity of the spectrum of a singular quasi-differential operator with respect to a parameter// Eurasian Math. J. 2:3, 67-81 (2011).

21. M.V. Fedoryuk. Asymptotics: integrals and series. Nauka, Moscow (1987). (in Russian).

22. H.B. Dwight. tables of integrals and other mathematical data. The Macmillan Company, New York (1961).

Khabir Kabirovich Ishkin,

Bashkir State University,

Zaki Validi str. 32,

450074, Ufa, Russia

E-mail: Ishkin62@mail.ru

Khairulla Khabibulovich Murtazin,

Bashkir State University,

Zaki Validi str. 32,

450074, Ufa, Russia

E-mail: Murtazin@mail.ru 